Pierric Giraud

Dominique Valade

Michel Lanteri-Minet

Anne Donnet

Gilles Geraud

Evelyne Guégan-Massardier

and the Observatoire des

Migraines et Céphalées of the

French Headache Society

\section{Is migraine with cranial nerve palsy an ophthalmoplegic migraine?}

Received: 27 November 2006

Accepted in revised form: 14 February 2007

Published online: 11 May 2007

P. Giraud ( $\square)$

Service de Neurologie A,

Centre Hospitalier de la région Annecienne,

1 Avenue du Tresum,

F-74000, Annecy, France

e-mail: pgiraud@ch-annecy.fr

Tel.: +33.450883337

Fax: +33.450883052

D. Valade

Centre Urgence Céphalées,

2 Rue Amboise Paré,

F-75475, Paris, France

M. Lanteri-Minet

Hôpital Pasteur, Département douleur,

30 avenue de la voie romaine,

F-06002, Nice Cedex 01, France

A. Donnet

Hôpital de la Timone,

Boulevard Jean Moulin,

Service de neurochirurgie,

F-13385, Marseille Cedex 5, France

G. Geraud

Hôpital Rangueil,

Service de neurologie,

1, Avenue J. Pouhles, TSA 50032,

F-31059, Toulouse Cedex 9, France

E. Guégan-Massardier

Département de neurologie,

CHU de Rouen F-76031 Cedex, France
Abstract Ophthalmoplegic migraine $(\mathrm{OM})$ is a rare form of primary headache. Because of its rarity, only a few cases, mostly symptomatic, are reported. We analyse nine cases among 52973 adults who suffer from headaches with an oculomotor palsy firstly considered as OM. The study was retrospective and multicentric in a database set up in France. The aim of our investigation was to describe the clinical and radiological aspects of these cases and to discuss the diagnosis of OM. We demonstrate that the characteristics of the headaches were identical to usual migraine without oculomotor nerve palsy for each case. The study emphasises the difficulty of the OM diagnosis even with the new IHS criteria because of the rarity of having all characateristics. A wide heterogeneity was noted in cranial imagery and blood tests. We suggest adding the code of probable OM in the IHS classification to increase the knowledge and detection of this type of headache. A biological blood test and an MRI are systematically required to help clinicians in their diagnosis and to exclude alternative aetiology of headache with palsy.

Keywords Ophtalmoplegic migraine $\cdot$ Cranial nerve palsy $•$ Migraine and ocular palsy 


\section{Introduction}

Ophthalmoplegic migraine (OM) was first described by Gubler in 1860 according to Pearce [1]. First considered as a clinical form of migraine, the second edition of the International Headache Disorders (ICHD II) classifies the disease as a cranial neuralgia and a central cause of facial pain, code 13-17 [2]. OM remains a rare disorder, with a prevalence, probably less than one case in a million [3]. In adults, this diagnosis is exceptional, because all alternative causes of painful oculomotor nerve palsy must first be excluded. To date, most of the reported cases are paediatric or even young adults [4]. The pathophysiology of the disease remains unknown. Recently, publications with cranial MRI depict the presence of contrast enhancement of the oculomotor nerve in the peri-cerebral region that is considered by authors as a specific sign [5]. However, this sign does not seem to be systematically present and thus was not included in the ICHD II criteria. In a retrospective study, we have identified nine cases of migraine with ophthalmoplegia belonging to the Observatoire des Migraines et Céphalées French database.

The aim of our study was to describe these cases and to discuss the concept and its limits in clinical practice.

\section{Patients and methods}

The French database (Observatoire des Migraines et Céphalées) is a French collaborative database set up by the French Headache Society (Société Française d'Etudes des Migraines et des Cephalées) in 2002. The database contains all headache patients who have consulted in 13 tertiary headache centres in France (Annecy, Bordeaux, Clermont-Ferrand, Colombes, Lille, Marseille, Nice, Paris, Toulouse, Tours, Rouen, Saint Etienne, Voiron) since October 2002. It is an adult database because no paediatric consultations are included in the project. All patients were classified in accordance with the ICHD I and secondly with the ICHD II criteria. On principle, when the disease was rare and even if all criteria were not present, the subject was included as a possible OM. This methodology was proposed to facilitate further study. With this method, we identified all patients with a diagnosis of OM included in the database from October 2002 to December 2005. The personal and family histories of headaches as well as clinical data from each case were collected. When available, the results of biological blood tests and brain imagery were collected.

\section{Results}

Among 52973 cases collected in the database, there were nine patients with OM diagnosis: six women and three males with an average age of 36 (16-74). The main data are summarised in Table 1. All cases suffered from migraine with (2) or without aura (7) that began before the first OM attack. Migraine was present in $60 \%$ of the families but in two cases this information was not available. OM had appeared either during childhood (1) or during adulthood (8). Headaches were present in seven cases before and during ophthalmoplegia (2 cases). The time between pain and ophthalmoplegia was variable (no time in two cases to 4 days). The description of the headaches was not different from usual migraine. Unilateral or frontal pulsating pain was systematically described. Its location was on the same side as the palsy. Attacks of ophthalmoplegia with pain were present only once in two cases whereas in seven cases several attacks were noted (two to more than four). The cranial nerve most commonly affected was the third one $(55 \%)$ but several times the palsy affected different nerves of the same patient (the third and the sixth nerve, 2 cases). In two cases, a diplopic sensation was described but no cranial nerve palsy was observed. In all cases, the general and neurological examination was always normal outside attacks. Biological blood test was very heterogeneous with wide exploration searching for diabetes mellitus, myasthenia or inflammatory disease in four cases. A brain CT-scan (one case) or MRI (seven cases) with digitalised angiography was performed except in one case. Brain CT-scan or MRI imagery was done once in seven cases. A single case had three CT-scans. The result was always normal or revealed no specific abnormalities such as abnormal white matter signals. No contrast enhancement was identified on cranial nerve but the delay between palsy and MRI was unknown in most cases. In no case was conventional angiography carried out. For the single case where no brain imagery was done, the repetition of attacks had first suggested a non-tumoral cause, but the patient was not followed up. Prophylactic or acute treatment was not systematically given and was varied as described in Table 1. The cranial palsy and headache was always reversible in a few weeks. The follow up was variable among centres. Two cases were lost to follow-up, and only one case presented another attack after inclusion in the database.

\section{Discussion}

This French retrospective multicentric study has identified nine cases of adults suffering from migraine with oculomotor nerve palsy in 3 years among 52973 headache patients seen in 13 tertiary centres. As the study is retrospective and multicentric, there is a heterogeneity in paraclinical examinations. However, the rarity of the $\mathrm{OM}$ 
Table 1 Main data of the ophtalmoplegic migrainer

\begin{tabular}{|c|c|c|c|c|c|c|c|c|c|}
\hline \multirow[t]{2}{*}{ Case } & \multirow{2}{*}{$\begin{array}{l}\text { Age (years), } \\
\text { sex }\end{array}$} & \multicolumn{3}{|c|}{ Ophtalmoplegic migraine } & \multirow[t]{2}{*}{ CT-Scan } & \multirow[t]{2}{*}{ MRI } & \multirow[t]{2}{*}{ Angiography } & \multirow[t]{2}{*}{ Treatment } & \multirow{2}{*}{$\begin{array}{l}\text { Evolution, } \\
\text { Duration of follow-up }\end{array}$} \\
\hline & & $\begin{array}{l}\text { Number } \\
\text { of attacks }\end{array}$ & $\begin{array}{c}\text { Time between } \\
\text { headache } \\
\text { and palsy }\end{array}$ & $\begin{array}{l}\text { Cranial } \\
\text { Nerve }\end{array}$ & & & & & \\
\hline 1 & $74, \mathrm{~F}$ & 3 & 4 days & III R, III, VI & $\mathrm{N}$ & $\mathrm{N}$ & $\mathrm{N}$ & No & NR, 3 years \\
\hline 2 & $48, \mathrm{~F}$ & 1 & Unknown & VI L & ND & $\mathrm{N}$ & $\mathrm{N}$ & Oxetorone & NR, 1 year \\
\hline 3 & $16, \mathrm{~F}$ & 1 & Unknown & III L & $\mathrm{N}$ & $\mathrm{N}$ & $\mathrm{N}$ & Not specify & NR, 2 years \\
\hline 4 & $16, \mathrm{M}$ & 3 & 2 & III & $\mathrm{N}$ & $\mathrm{N}$ & $\mathrm{N}$ & Not specify & $\mathrm{NR}, 2$ years \\
\hline 5 & $54, \mathrm{M}$ & 2 & 5 & VI R, VI L & $\mathrm{N}$ & $\mathrm{N}$ & $\mathrm{N}$ & Oxetorone & NR, 4 years \\
\hline 6 & $23, \mathrm{~F}$ & 4 & No delay & III L & ND & ND & ND & NSAiDS & Lost sight \\
\hline 7 & $29, \mathrm{M}$ & $>5$ & $30 \min$ & diplopia & $\mathrm{N}$ & $\mathrm{N}$ & $\mathrm{N}$ & Oxetorone, & NR, 1 year \\
\hline 8 & $24, \mathrm{~F}$ & 2 & No delay & diplopia & ND & $\mathrm{N}$ & $\mathrm{N}$ & Not specify & Lost sight \\
\hline 9 & $48, \mathrm{~F}$ & 4 & No delay & III L & ND & $\mathrm{N}$ & $\mathrm{N}$ & NSAiDS & $\mathrm{R}, 1$ year \\
\hline
\end{tabular}

$F$, female; $M$, male; $I I I$, oculomotor nerve; $V I$, abducens nerve; $N$, normal; $N D$, not done; $L$, left; $R$, right; $N R$, no recurrence; $R$, recurrence

observed is in accordance with previous work that underlines that $\mathrm{OM}$ is exceptional in the general population [4]. For instance, the prevalence of OM in Scandinavia was of 0.7 per million in a previous study in the general population [3]. On the contrary, Donahue reported $14 \%$ of diplopia among 1600 cases of migraine sufferers [6]. This high prevalence of an ophthalmic symptom was never demonstrated in other studies. The discrepancy among studies can be explained by the limited size of the Donahue study [6].

Several data of OM are underlined in our work even though it is more complex to analyse data from a retrospective analysis than a prospective study. On clinical grounds, we can insist on several aspects of the disease found routinely. Firstly, OM remains a diagnosis of exclusion in clinical practice even if the ICHD II proposes several strict criteria to identify the disease [2]. These criteria are mainly based on clinical signs and repetition of the attacks without abnormality in paraclinical examinations. In ICHD II, headache is described as migraine-like but in most published cases, the description is non-existent or poor [4-7]. In fact, in our cases the description of pain does not appear different in type, localisation or intensity from usual migraine attacks. Therefore, this work demonstrates that pain in OM is very similar to other migraine attacks without ophthalmoplegia. Secondly, by definition, headaches accompany or follow within the 4 days of its onset by paralysis of one or more of the third, fourth and sixth cranial nerves. Østergaard et al. [8] reported a single unusual case where four attacks of ophthalmoplegia were present without headache in a baby. Unilateral headache was present only at the fifth episode at the age of three. This exceptional case puts forward the question of accept- able time between palsy and pain. In order to improve the distinction between OM and other causes of nerve palsy, ICHD II proposes a maximum time of 4 days between the two signs [2]. This time, in light of our work, is helpful in clinical practice because all cases are in accordance with this criterion. In two of our cases headache appeared simultaneously with cranial palsy, as reported by Lance and Zagani [7]. In these cases, the clinical signs as well as the spontaneous good evolution without corticosteroid rule out the diagnosis of Tolosa-Hunt syndrome. Thirdly, repetition of cranial nerve palsy is necessary for the definite diagnosis of OM. Two of our cases have had only one episode of cranial nerve palsy with migraine headache and should theoretically not be classified as OM in ICHD II. The duration of the follow-up for these two cases was one and two years. No recurrence was observed. As no alternative aetiologies were present, they were coded as OM. Because the situation is also not exceptional, IHS classification would offer the possibility to code them as probable OM. Probable OM could be proposed as probable migraine in cases where attacks and/or headache without one of the features needed to fulfil all criteria for OM. This possibility would improve the detection of OM. Fourthly, the oculomotor nerve is mostly implicated in $\mathrm{OM}[3,4,7,8]$. However, this nerve is concerned in $44 \%-66 \%$ in acute ocular mononeuropathy in the general population. Periorbital pain is also almost systematically depicted whatever the cause in this situation $[9,10]$. A microvascular ischaemia of the pericerebral portion of the nerve is frequently suspected, explaining both signs but other causes such as a viral infection or a metabolic abnormality is possible. In OM, the pathophysiological mechanism remains debated but our work brings no information 
on this aspect because it is an observational study. The main hypothesis is that $\mathrm{OM}$ is due to a demyelinating neuropathy [7]. This hypothesis is supported by the presence of a contrast enhancement and a thickening of the ocular nerve in its cisternal portion detected by MRI that was not observed in our cases $[4,5]$. The average duration of this radiological abnormality is 7-9 weeks, although few studies are available on this topic [5]. Nevertheless, this sign is not systematically observed, which allows other hypotheses. Mark et al. [5] have suggested that a benign viral or an idiopathic inflammatory neuropathy can be implicated because the contrast enhancement of the nerve could be observed in such cases.

In the literature, the research for an alternative cause is particularly heterogeneous, as observed in our work. Because numerous diseases can mimic OM, such as vascular aneurysm, myasthenia gravis, oculomotor nerve schwannoma, pituitary apoplexy, Tolosa-Hunt syndrome, mucomycosis and ischaemic or idiopathic cranial palsy, paraclinical examinations are always required $[4,5]$. In published cases as in ours, biological blood tests are sometimes carried out to rule out diabetes mellitus, myasthenia gravis or an inflammatory disease [3, 4, 11]. To date, there is no consensus on the tests and ICHD II does not recommend making such biological investigations and gives no guidelines for blood tests. As for clinical description, future recommendation would propose the type and number of biological blood tests necessary for OM diagnosis. On the contrary, ICHD II recommends ruling out by appropriate investigation parasellar, orbital fissure or posterior fossa lesion. The choice of radiological examination is also not specified, allowing clinicians to do CT-scan or MRI. This heterogeneity is well represented in our observation, coming from several six different centres. Because of the presence of a possible contrast enhancement of the cranial nerve and the greater sensitivity of MRI, this radiological exploration should be systematically proposed to exclude the main differential diagnosis in the future $[3,6$, $12,13]$.

Our retrospective study emphasises the difficulties of making an OM diagnosis and demonstrates the heterogeneity of the paraclinical tests performed for giving such a diagnosis. Although clinical signs are now well described in ICHD-II, diagnosis remains difficult in clinical practice because numerous diseases can produce migraine and oculomotor nerve palsy. This work suggests that the future IHS guidelines insist on a biological blood test as well as the type of cranial imagery necessary for OM diagnosis. Cranial MRI with contrast appears fundamental not only to exclude an alternative aetiology but also to improve the knowledge of this rare disease. Another proposition should be to have the possibility to identify a case as probable OM when all criteria are not present, in order to follow the evolution of this rare disease.

Acknowledgement This study was funded by a grant from GlaxoSmithKline.

\section{References}

1. Pearce $\mathbf{J}$ (1968) The ophthalmological complication of migraine. J Neurol Sci 6:73-81

2. Headache Classification SubCommittee of the International Headache Society. (2004) The International Classification of Headache Disorder, 2nd edn. Cephalalgia 24:9-160

3. Hansen SL, Borelli-Moller L, Strange P et al (1990) Ophthalmoplegic migraine: diagnostic criteria, incidence of hospitalization and possible etiology. Acta Neurol Scand 81:54-60

4. De Silva DA, Siow HC (2005) A case report of ophthalmoplegic migraine: a differential diagnosis of third nerve palsy. Cephalalgia 25:827-830
5. Mark AS, Casselman J, Brown D et al (1998) Ophthalmoplegic migraine: reversible enhancement and thickening of the cisternal segment of the oculomotor nerve on contrast-enhanced MR images. Am J Neuroradiol 19:1887-1891

6. Donahue HC (1949) Migraine and its ocular manifestations. Trans Am Ophthalmol Soc 47:554-605

7. Lance JW, Zagani AS (2001) Ophthalmoplegic migraine: a recurrent demyelinating neuropathy? Cephalalgia 21:84-89

8. Østergaard JR, Møller HU, Christensen $\mathrm{T}$ (1996) Recurrent ophthalmoplegia in childhood: diagnostic and etiologic considerations. Cephalalgia 16:276-279
9. Berlit P (1991) Isolated and combined pareses of cranial nerves III, IV and VI. A retrospective study of 412 patients. J Neurol Sci 103:10-15

10. Chou KL, Galetta SL, Liu GT et al (2004) Acute ocular motor mononeuropathies: prospective study of the roles of neuroimaging and clinical assessment. J Neurol Sci 219:35-39

11. Murakami T, Funatsuka M, Komine M et al (2005) Oculomotor nerve schwannoma mimicking ophthalmoplegic migraine. Neuropediatrics 36:395-398

12. Doran M, Larner AJ (2004) MRI findings in ophthalmoplegic migraine: nosological implications. J Neurol 251:100-101

13. Lee TG, Choi WS, Chung KC (2002) Ophthalmoplegic migraine with reversible enhancement of intraparenchymal abducens nerve on MRI. Headache 42:140-141 